\title{
Néel-Dimer Transition in Antiferromagnetic Heisenberg Model and Deconfinement of Spinons at the Critical Point
}

\author{
Daisuke Yoshioka, Gaku Arakawa and Ikuo Ichinose \\ Department of Applied Physics, Graduate School of Engineering, \\ Nagoya Institute of Technology, Nagoya, 466-8555 Japan \\ Tetsuo Matsui \\ Department of Physics, Kinki University, Higashi-Osaka, 577-8502 Japan
}

(September 17, 2018)

\begin{abstract}
Quantum phase transition from the Néel to the dimer states in an antiferromagnetic(AF) Heisenberg model on square lattice is studied. We introduce a control parameter $\alpha$ for the exchange coupling which connects the Néel $(\alpha=0)$ and the dimer $(\alpha=1)$ states. We employ the $C P^{1}$ representation of the $s=\frac{1}{2}$ spin operator and integrate out the half of the $C P^{1}$ variables at odd sites to obtain a $C P^{1}$ nonlinear $\sigma$ model. The effective coupling constant is a function of $\alpha$ and at $\alpha=0$ the $C P^{1}$ model is in the ordered phase which corresponds to the Néel state of the AF Heisenberg model. A phase transition to the dimer state occurs at a certain critical value of $\alpha_{C}$ as $\alpha$ increases. In the Néel state, the dynamical composite $\mathrm{U}(1)$ gauge field in the $C P^{1}$ model is in a Higgs phase and low-energy excitations are gapless spin wave. In the dimer phase, a confinement phase of the gauge theory with $s=1$ excitations is realized. For the critical point, we argue that a deconfinement phase, which is similar to the Coulomb phase in 3 spatial dimensions, is realized and $s=\frac{1}{2}$ spinons appear as low-energy excitations.
\end{abstract}

Quantum phase transition(QPT) is one of the most interesting problem in these days. ${ }^{1}$ It is often argued that the simple Ginzburg-Landau theory does not apply to certain class of the QPT's. In this paper we shall study $s=\frac{1}{2}$ antiferromagnetic(AF) Heisenberg model on 2-dimensional square lattice with nonuniform exchange couplings,

$$
H_{A F}=\sum_{x, j} J_{x j} \vec{S}_{x} \cdot \vec{S}_{x+j}
$$

where $x$ denotes site of the spatial lattice, $j$ is the direction index $(j=1,2)$ and $\vec{S}_{x}$ is the spin operator at site $x$. We rename the even lattice sites $x=(o, i)$ where $o$ denotes odd site and the index $i=1,2,3$ and 4 specifies its four nearest-neighbor(NN) even sites (see Fig.1).
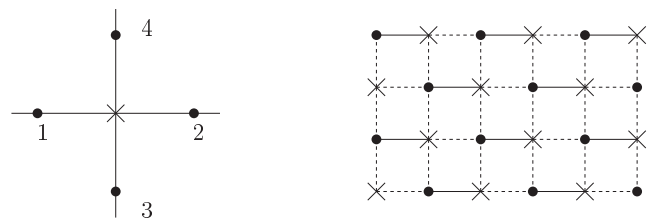

FIG. 1. Numbering and dimer picture:cross symbol is odd site, and spot symbol is even site. Solid line means that its correlation is stronger than dotted lines.

The exchange couplings $J_{x j}=J_{o i}$ are position dependent and we explicitly consider the following case which corresponds to the dimer configuration,

$$
\begin{aligned}
J_{o i} & =J+\Delta J_{o i}, \\
\Delta J_{o i} & = \begin{cases}\Delta J_{o i}=\alpha J, & i=1 \\
\Delta J_{o i}=-\alpha J, & i=2,3,4\end{cases}
\end{aligned}
$$

where $0 \leq \alpha \leq 1$ is a control parameter which connects the uniform Heisenberg model to the dimer model.

It is not so difficult to derive the $C P^{1}$ field-theory model $^{2,3}$ from Eq. $(1)^{4}$. The spin operator $\vec{S}_{x}$ can be expressed in terms of the $C P^{1}$ variable $z_{x}=\left(z_{x}^{1}, z_{x}^{2}\right)^{t}$ as

$$
\vec{S}_{x}=\frac{1}{2} z_{x}^{\dagger} \vec{\sigma} z_{x},
$$

where $\vec{\sigma}$ are the Pauli matrices and the $C P^{1}$ constraint $\sum_{a=1,2}\left|z_{x}^{a}\right|^{2}=1$ guarantees the magnitude of the localized spin as $\frac{1}{2}$.

From our assignment of $J_{o i}(2)$, it is obvious that $J_{o 1}$ is larger than the others. We use the path-integral formalism and parameterize the $C P^{1}$ variable $z_{o}$ by refering to $z_{o 1} \equiv z_{e}$,

$$
z_{o}=p_{o} z_{e}+\sqrt{1-\left|p_{o}\right|^{2}} e^{i \theta} \tilde{z}_{e},
$$

where $p_{o}$ is a parameter, $e^{i \theta}$ is a phase factor and $\tilde{z}_{e}=i \sigma_{2} z_{e}^{*}$. At vanishing temperature $(T)$, spins tend to point antiparallel their NN spins, and then the parameter $p_{o}$ can be treated as a small parameter. We expand $\sqrt{1-\left|p_{o}\right|^{2}} \simeq 1-\frac{1}{2}\left|p_{o}\right|^{2}+\cdots$ and retain only terms up to quadratic of $p_{o}$. Then we perform the Gaussian integration of $p_{o}$ 's to obtain an effective model of $z_{e}$ 's for which smooth configurations dominate at $T=0$.

Calculation is rather long but straightforward ${ }^{4}$ and we obtain an effective field theory of the AF Heisenberg model under study,

$$
\mathcal{L}_{C P}=\sum_{\mu=\tau, x}\left[\left|D_{\mu} z_{x}\right|^{2}+\sigma_{x}\left(\left|z_{x}\right|^{2}-\frac{1}{f_{\mathrm{eff}}}\right)\right]+\mathcal{L}_{\mathcal{B}},
$$


where $D_{\mu} z_{x}=\left(\partial_{\mu}+i A_{\mu}\right) z_{x}, A_{\mu}=i z^{\dagger} \partial_{\mu} z$ is the $\mathrm{U}(1)$ gauge field, $\sigma_{x}$ is the Lagrange multiplier for the $C P^{1}$ constraint and $\mathcal{L}_{\mathcal{B}}$ is the Berry phase term. We have rescaled the imaginary time $\tau$ and $x$ as taking the continuum limit(the spatial coordinate $x$ in Eq.(5) denotes the even site of the original lattice). The effective coupling constant $f_{\text {eff }}$ and the "speed of light" $c$, which is often set unity, are explicitly given as

$$
\begin{aligned}
\frac{1}{f_{\mathrm{eff}}} & =\frac{1}{2 \sqrt{2} a} \cdot \frac{1-\alpha}{2-\alpha} \sqrt{\frac{2(2+\alpha)}{1-\alpha}} \equiv \frac{1}{f_{\mathrm{AFH}}} \frac{1}{b(\alpha)}, \\
c & =\frac{\sqrt{2} a J}{\hbar} \cdot \sqrt{\frac{(2-\alpha)(1-\alpha)}{2}} \equiv c_{\mathrm{AFH}} \gamma(\alpha),
\end{aligned}
$$

where $a$ is the lattice spacing of the original lattice and $f_{\mathrm{AFH}}=2 \sqrt{2} a$ and $c_{\mathrm{AFH}}=\frac{\sqrt{2} a J}{\hbar}$.

Here we should comment on the Berry phase terms, $\mathcal{L}_{\mathcal{B}}$, which appear in the $C P^{1}$ nonlinear- $\sigma$ model representation of the AF Heisenberg model. First we consider $\mathrm{AF}$ spin chains in one dimension in order to obtain important insight for the effect of the Berry phase terms. For the uniform AF chains, the Berry phase reduces to the $\theta$-term $\left(i \theta \int d \tau d x F_{\tau x}\right)$ with $\theta=\pi$. The gauge dynamics crucially depends on the value of $\theta$ and only the case of $\theta=\pi(\bmod 2 \pi)$, the deconfinement phase is realized. Topologically nontrivial configurations are suppressed by the $\theta$-term when $\theta=\pi$.

On the other hand for the bond-alternating(BA) AF Heisenberg chain with $J_{x}=J+(-1)^{x} \Delta J$, the value of the $\theta$ parameter is $\theta=\frac{J-\Delta J}{J+\Delta J} \pi$, and therefore the above suppression by the $\theta$-term does not occur and gauge dynamics is essentially the same with the $\theta=0$ case. Then the confinement of the spinon $z_{x}^{a}$ occurs, and low-energy excitations are gapful $s=1$ excitations $\vec{n}_{x}=\frac{1}{2} z_{x}^{\dagger} \vec{\sigma} z_{x}$ even for a very small value of $\Delta J$. This is nothing but the spin-Peierls transition.

For the 2-dimensional uniform AF Heisenberg model, the Berry phase term similarly appears as $\mathcal{L}_{\mathcal{B}}=$ $\frac{i}{2} \sum_{x} \epsilon_{x} \mathcal{A}_{x}$, where $\epsilon_{x}=1(-1)$ for even (odd) site $x$ (of the lattice with the lattice spacing $\sqrt{2} a$ for the $C P^{1}$ model (5)) and $\mathcal{A}_{x}$ is the area enclosed by the curve given by the time evolution of $\vec{n}_{x}(\tau)$. It can be proved that it gives only vanishing contribution for an arbitrary smooth configuration. However for singular configurations like instantons (monopoles), the Berry phase is nonvanishing and it gives terms like $\mathcal{L}_{\mathcal{B}}=i \frac{\pi}{2} \sum_{i=1}^{4} \sum_{x} \zeta_{x}^{i} m_{x}^{i}$, where $\zeta_{x}^{i}$ are $0,1,2,3(i=1,2,3,4)$, respectively and $m_{x}^{i}$ is the instanton number. ${ }^{5,6}$ (The index $i$ refers to the four dual sublattices of the original lattice.) From the above $\mathcal{L}_{\mathcal{B}}$, it is obvious that the cancellation mechanism of the instanton contribution occurs by the destructive interference unless $m_{x}^{i}=4(\bmod 4)$.

For the nonuniform cases which are considered in this paper, the coefficient of the Berry phase term becomes irrational as in the case of the BA spin chains and therefore cancellation of instanton effect does not occur or at least weakens. Then we can expect that the (global) phase structure of the effective gauge model for the nonuniform AF Heisenberg model is the same with that of the $C P^{1}$ model without the Berry phase terms. More comments will be given after showing results of nonperturbative studies on the gauge dynamics.

It is straightforward to obtain the effective potential of $\sigma=\left\langle\sigma_{x}\right\rangle$ and $z^{2}=\left\langle z_{x}^{2}\right\rangle$ by integrating out $z_{x}^{1}$,

$V_{\mathrm{eff}}=\sigma\left(\left|z^{2}\right|^{2}-\frac{1}{f_{\mathrm{eff}}}\right)+\frac{1}{6 \pi}\left[\left(\sigma+\Lambda^{2}\right) \sqrt{\sigma+\Lambda^{2}}-\Lambda^{3}-\sigma \sqrt{\sigma}\right]$,

where the cutoff $\Lambda=\frac{\sqrt{2 \pi}}{a}$. The effective potential $V_{\text {eff }}$ indicates that there exists a critical coupling $f_{C}=\frac{4 \pi}{\Lambda}$. The existence of the phase transition has been verified by the numerical calculation of the equivalent $O(3)$ nonlinear $\sigma$ model in $(2+1)$ dimensions $^{7}$. In the weak-coupling region $f_{\text {eff }}<f_{C}$, the spontaneous symmetry breaking occurs and $\left\langle z_{x}^{2}\right\rangle \neq 0$. As a result, the Higgs phase is realized in the gauge-theory terminology. Low-energy excitations are gapless spin waves which are described by $z_{x}^{1}$. In the strong-coupling phase $f_{\text {eff }}>f_{C},\left\langle\sigma_{x}\right\rangle \neq 0$ whereas $\left\langle z_{x}^{2}\right\rangle=0$. Local Maxwell terms appear in the effective action of the gauge field $A_{\mu}$, and the confinement phase is realized. Low-energy excitations are $s=1$ composite of the spinons which correspond to $\vec{n}_{x}$ with (mass) ${ }^{2}$ $\propto\left\langle\sigma_{x}\right\rangle$.

The effective coupling $f_{\text {eff }}$ in (6) first decreases as $\alpha$ increases but above certain value of $\alpha$ it starts to increase and goes to infinity at the dimer limit $\alpha=1$. In the uniform case $\alpha=0, f_{\text {eff }}(\alpha=0)<f_{C}$ and this means that the ordered Néel state is realized at the vanishing $T$ in the AF Heisenberg model in two spatial dimensions as it is now widely believed. The behavior of the effective coupling $f_{\text {eff }}(\alpha)$ shows the existence of a critical value $\alpha_{C}$ at which the phase transition occurs. This result indicates that the strong-coupling phase of the $C P^{1}$ model (5) corresponds to the dimer phase in which the ground state is nothing but spin-singlet pairs formed by the alternative strong exchange couplings and excitations have $s=1$. In fact this Néel-dimer transition was observed by the numerical calculations some years ago ${ }^{8}$.

Hereafter we are interested in the critical point at $f_{\text {eff }}=f_{C}$ which separates the Néel and dimer phases. In order to investigate that "phase", study on the gauge dynamics is required. At $f_{C},\left\langle\sigma_{x}\right\rangle=\left\langle z_{x}^{a}\right\rangle=0$. Effective action of the gauge field and the field $\sigma_{x}$ is obtained by integrating out the gapless spinon field $z_{x}^{a}(a=1,2)$. The resultant effective action becomes nonlocal and therefore it is possible for the gauge dynamics to belong to different universality class from that of the usual gauge theory in $2+1$ dimensions. By the continuum field-theory calculation, the effective action of the gauge field $A_{\mu}$ is obtained as

$$
\mathcal{L}_{A} \propto \int d^{3} x \int d^{3} y \sum_{\mu, \nu} F_{\mu \nu}(x) \frac{1}{|x-y|^{2}} F_{\mu \nu}(y),
$$

where $F_{\mu \nu}=\partial_{\mu} A_{\nu}-\partial_{\nu} A_{\mu}$. Similarly the effective action of the field $\sigma_{x}$ is obtained as 


$$
\mathcal{L}_{\sigma} \propto \int d^{3} p \tilde{\sigma}(-p) \frac{1}{|p|} \tilde{\sigma}(p),
$$

where $\tilde{\sigma}(p)$ is the Fourier transformed field of $\sigma_{x}$. Equation (9) shows that fluctuations of the field $\sigma_{x}$ are strongly suppressed at large distances. In the $C P^{1}$ model on the 3 -dimensional space-time cubic lattice, a similar expression of the action $\mathcal{L}_{A}$ and $\mathcal{L}_{\sigma}$ is obtained by the hopping expansion of $z_{x}^{a}(a=1,2)$. In the effective action $S_{A}$ of the compact gauge field $U_{\mu}=e^{i A_{\mu}}$, the following nonlocal terms appear

$$
S_{A} \sim \sum_{\Gamma} \gamma^{|\Gamma|} \prod_{\Gamma} U_{\mu}(x)
$$

where the summation over closed loops $\Gamma$ includes loops of an arbitrary large size, $|\Gamma|$ is the length of $\Gamma$ and the parameter $\gamma$ is estimated as $\gamma \sim \frac{1}{2 d}$ for massless $z_{x}^{a}$ with the dimension of the lattice $d=3$. We shall focus our interest on the gauge dynamics of the above nonlocal action which is one of the most important problems in the theoretical studies on the strongly-correlated electron systems in these days and is still controversial. At present it is known that there exists only the confinement phase in the $(2+1)$-dimensional compact $\mathrm{U}(1)$ gauge theory without matter couplings. ${ }^{9}$ However phase structure is not clear when the $\mathrm{U}(1)$ gauge field couples to matter fields, particularly gapless matter fields ${ }^{10-14}$. In particular in Ref. ${ }^{10,11}$, it is argued that a deconfinement phase is realized by the gapless fermion couplings. Simple mean-field type argument is not applicable for the nonlocal gauge systems and numerical studies are required.

We shall study lattice gauge model with a nonlocal action which is related with (10) but slightly more tractable. The summation over $\Gamma$ in $S_{A}(10)$ becomes (logarithmically) divergent for the configulation $U_{\mu}=1^{15}$ since the massless relativistic bosons $z_{x}^{a}(a=1,2)$, which appear at the critical point, give divergent hopping expansion for $U_{\mu}=1$. From the above discussion we shall consider the following $(2+1)$ dimensional lattice gauge model with the long-range interaction in the $\hat{\tau}$ direction,

$$
\begin{aligned}
S_{G}= & g_{1} \sum_{n=1}^{N_{\tau}-1} \sum_{x, \mu=1,2} \frac{1}{n} U_{\mu}(x) W_{x+\mu}(n) U_{\mu}^{\dagger}(x+n \hat{\tau}) W_{x}^{\dagger}(n) \\
& +g_{2} \sum_{p l} \prod_{p l} U
\end{aligned}
$$

where $W_{x}(n)=U_{0}(x) U_{0}(x+\hat{\tau}) \cdots U_{0}(x+n \hat{\tau}), N_{\tau}$ is the system size in the $\hat{\tau}$ direction and $g_{i}(i=1,2)$ are coupling constants for the time and spatial directions, respectively. From action (11), it is obvious that the gauge model under study has nonlocal coupling in the $\hat{\tau}$ direction whereas it has the usual local Maxwell-type correlation in the spatial directions. ${ }^{16}$ Reason why we take the action (11) is that the $\hat{\tau}$ direction terms logarithmically divergent for $U_{\mu}=1$ and also Monte-Carlo simulations are easier for the model than those with full-nonlocal interaction terms. We think that studies of the model (11) give important insight for the full-nonlocal gauge system (10). More comments on this point will be given after showing the results of the Monte-Carlo simulations of the model (11).

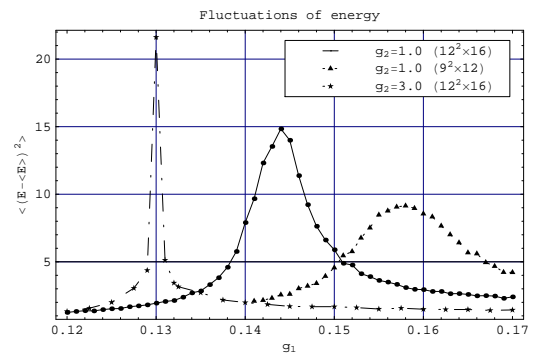

FIG. 2. Fluctuation of energy as a function of $g_{1}$ is plotted for the fixed $g_{2}=1.0$ and 3.0. Lattice size is $12^{2} \times 16$ and $9^{2} \times 12$. The results show the existence of a phase transition.
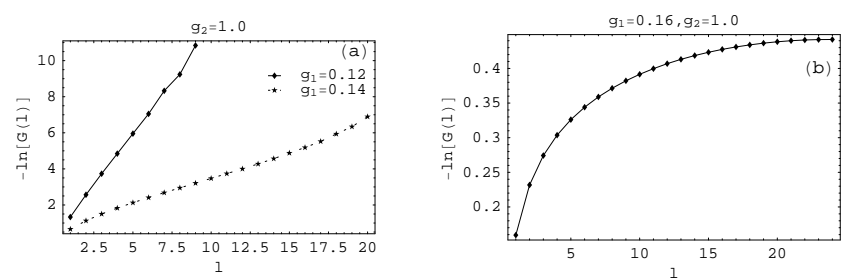

FIG. 3. The correlations of Polyakov lines as functions of their distance are plotted. The results for $g_{1}=0.12$ and 0.14 are shown in (a), and one for $g_{1}=0.16$ is shown in (b). Value of $g_{2}$ is fixed as $g_{2}=1.0$. The results show the existence of the confinement-deconfinement phase transition.

We studied phase structure of the model (11) by the Monte-Carlo simulations of the standard Metropolis algorithm. We calculated the fluctuation of the energy $E$, i.e., $\left\langle(E-\langle E\rangle)^{2}\right\rangle$, as a function of the coupling $g_{1}$ for fixed $g_{2}$. The results are shown in Fig. 2 for $g_{2}=1.0$ and $g_{2}=3.0$, and they indicate the existence of a phase transition at critical coupling $g_{1 C} \sim 0.14$ and $g_{1 C} \sim 0.13$, respectively. We varied the system size and verified that the peak gets sharper as the system size increases. This means that the transition is of second order. In order to investigate gauge dynamics in each phase we calculated the correlations of the Polyakov lines $G(\ell)=\left\langle P(x) P^{\dagger}(x+\ell \hat{1})\right\rangle$ where $P(x)=U_{0}(x) \cdots U_{0}\left(x+N_{\tau} \hat{\tau}\right) .{ }^{17}$ In Fig. 3, we show the results of $G(\ell)$ for various values of $g_{1}$ and it is obvious that the behavior of $G(\ell)$ changes at $g_{1} \sim g_{1 C}$ from $\ln G(\ell) \propto \ell$ to $G(\ell) \sim$ constant for large $\ell$. Then the phase transition from the confinement to the deconfinement phases occurs as $g_{1}$ increases.

Numerical calculations show that the spatial coupling of the gauge field and larger system size enhance the deconfinement phase. From the above results we expect that the full-nonlocal gauge model (10), which results from the integration of the massless relativistic boson $z_{x}^{a}$ at the criticality, is in the deconfinement phase. In fact 
the coupling constant $g_{1} \sim 1$ corresponds to (10) in which the damping factor $\gamma^{|\Gamma|}$ balances the entropy factor of the paths $\Gamma$.

In the deconfinement phase of (11), topologically nontrivial configurations are suppressed and the field-theory result (8) gives a qualitatively correct picture. Charges interacting through $A_{\mu}$ have the potential $V(r) \propto 1 / r$ where $r$ is the spatial distance between the two charges.

Let us comment on the effects of the Berry phase. Since $\mathcal{L}_{B}$ is neglected in (11), one may doubt the deconfinement phase transition observed above. However, as the Berry phase generates extra phases for topologically nontrivial configurations in the path integral, the Berry phase enhances the deconfinement. In fact without these extra phases, all instanton configurations contribute additively to disorder the gauge system. Thus the existence of the deconfinement phase in the gauge system (11) guarantees its existence even in the presence of the Berry phase. Similar argument was used for the deconfinement transition at finite $T^{18}$, which is established at present.

We summarize the phase structure of the original spin model. In the region $\alpha<\alpha_{C}\left(f_{\text {eff }}<f_{C}\right)$, the low-energy excitations are the massless spin waves whereas in the region $\alpha>\alpha_{C}\left(f_{\text {eff }}>f_{C}\right)$, they are $s=1$ excitations $\vec{n}_{x}$. On the critical point $\alpha=\alpha_{C}\left(f_{\text {eff }}=f_{C}\right)$, the gauge dynamics is in the "Coulomb" phase and the low-energy excitations are the $s=1 / 2$ bosonic spinons $z_{x}^{a}(a=1,2)$ which are interacting with each other by the potential $1 / r$. The spin correlation function decays algebraically both in the Néel state and at the criticality but exponent is different. In the Néel state, the spin operator is given as $\vec{S}_{x}=\frac{1}{2} z_{x}^{\dagger} \vec{\sigma} z_{x} \sim\left\langle z_{x}^{\dagger}\right\rangle \vec{\sigma} z_{x} \sim z_{x}^{1}$ whereas at the criticality $\vec{n}_{x}$, the bilinear of $z_{x}^{a}$ and $z_{x}^{a \dagger}(a=1,2)$. Phase structure of the nonuniform AF Heisenberg model is schematically shown in Fig.4.

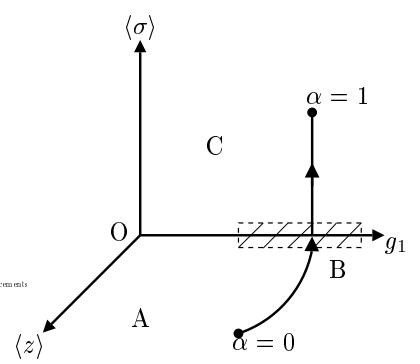

FIG. 4. Schematic phase diagram of the Heisenberg model. Phases A,B and C are the Higgs (Néel), Coulomb (critical) and confinement (dimer) phases, respectively.

Recently a similar phase transition from the Néel to the dimer states was discussed ${ }^{19}$. There they conclude that instanton effects are irrelevant at the critical point. Our numerical investigation is consistent with their result but our study shows the long-range interactions of the gauge field, which appear as a result of the coupling to the massless boson $z_{x}^{a}$, play an essentially important role. Results of more detailed studies on the long-range gauge theories will be reported in near future. ${ }^{20}$

${ }^{1}$ See for example, S.Sachdev, "Quantum Phase Transitions", (Cambridge University Press, Cambridge, England, 1999).

2 A.D'Adda, P.Di Vecchia and M. Lüscher, Nucl.Phys.B146, 63(1978); E.Witten, Nucl.Phys.B149, 285(1979).

${ }^{3}$ I.Ya. Aref'eva and S.I.Azakov, Nucl.Phys.B162, 298(1980).

${ }^{4}$ For the $s=\frac{1}{2}$ AF Heisenberg models, standard derivations were given by I.Ichinose and T.Matsui, Phys.Rev.B45, 9976(1992); Phys.Rev.B57, 13790(1998); See also D.P. Arovas and A. Auerbach, Phys.Rev.B38, 316(1988).

${ }^{5}$ F.D.M.Haldane, Phys.Rev.Lett.61, 1029(1988).

${ }^{6}$ N.Read and S.Sachdev, Phy.Rev.Lett.62, 1694(1989); Phys.Rev.B42, 4568(1990).

${ }^{7}$ See for example, M.Kamal and G.Murthy, Phys.Rev.Lett.71, 1911(1993) and references cited therein.

${ }^{8}$ P.Tomczak and J.Richter, J.Phys.A34, L461(2001) and references cired therein; see also R.R.P.Singh, M.P.Gelfand, and D.A.Huse, Phys.Rev.Lett.61, 2484(1988).

${ }^{9}$ A.M.Polyakov, Nucl.Phys.B120, 429(1977).

${ }^{10}$ I.Ichinose and T.Matsui, Phys.Rev.Lett.86, 942(2001).

${ }^{11}$ I.Ichinose and T.Matsui, and M.Onoda, Phys.Rev.B64, 104516(2001).

12 H.Kleinert, F.S.Nogueira, and A.Sudbø, Phys.Rev.Lett.88, 232001(2002); Nucl.Phys.B666, 361(2003).

13 I.F.Herbut and B.H.Seradjeh, Phys.Rev.Lett.91, 171601 (2003).

${ }^{14}$ I.F.Herbut, B.H.Seradjeh, S.Sachdev, and G.Murthy, Phys.Rev.B68, 195110 (2003).

${ }^{15}$ In the strong-coupling phase, the field $z_{x}$ acquires nonvanishing mass by $\left\langle\sigma_{x}\right\rangle \neq 0$. In this case, the summation over $\Gamma$ is convergent even for $U_{\mu}=1$.

16 The model (11) can be extended for the strong-coupling phase in which $\left\langle\sigma_{x}\right\rangle \neq 0$. The nonlocal terms become as $\sum_{n=1} \frac{1}{n} e^{-|\langle\sigma\rangle| n} U_{\mu}(x) W_{x+\mu}(n) U_{\mu}^{\dagger}(x+n \hat{\tau}) W_{x}^{\dagger}(n)$.

17 We calculate the correlation of the Polyakov lines instead of the Wilson loop since the system is anisotropic. The spatial correlation of the Polyakov lines can be an order parameter of the deconfinement transition in the gauge system (11).

18 A.M.Polyakov, Phys.Lett.B72, 477(1978); L.Susskind, Phy.Rev.D20, 2610(1979).

19 T.Senthil, L.Balents, S.Sachdev, V.Vishwanath, and M.P.A.Fisher, cond-mat/0312617, "Quantum criticality beyond the Landau-Ginzburg-Wilson paradigm".

${ }^{20}$ G.Arakawa, I.Ichinose, T.Matsui, and K.Sakakibara, work in progress. 